\title{
Klarheit schaffen, Herausforderungen der Zukunft annehmen und maßgeschneidert behandeln
}

\section{Blickdiagnosen \\ Welche Diagnose vermuten Sie? FOKUS}

Eine 34-jährige Frau stellte sich in unserer Notaufnahme mit tiefgreifender, selbst in Ruhe vorliegender Dyspnoe in Verbindung mit Orthopnoe, Ödem der unteren Extremitäten und Gewichtszunahme vor. Die Luftnot war ihr vor einem Jahr bei körperlicher Anstrengung erstmals aufgefallen. Kurz zuvor, im Dezember 2009, war ihr die Gabe von zusätzlichem Sauerstoff über eine Nasenbrille verschrieben worden.

Ihre medizinische Vorgeschichte war komplex und in Bezug auf folgende Befunde bedeutsam: (1) rezidivierende Pankreatitis, (2) eine minimal funktionsfähige linke Niere infolge einer chronischen perinephritischen Entzündung unklarer Ätiologie; (3) Visusminderung mit Stauungspapille, was eine beidseitige Dekompression der Optikusscheide im Juli 2008 erforderlich machte, und (4) autoimmunhämolytische Anämie nach Erythrozytentransfusion. Bei ihrer Vorstellung hatte die Patientin einen Blutdruck von 152/84 mmHg und eine Herzfrequenz von 60 Schlägen/min. Ihre
Atemfrequenz lag bei 28 Atemzügen/min., und ihre Sauerstoffsättigung betrug $80 \%$ bei 4 Litern Sauerstoff pro Minute über die Nasenbrille. Sie war adipös und wies im unteren Bereich des rechten Augenlids ein Xanthelasma auf. Eine Abklärung in Bezug auf eine Distension der Jugularvene war uns nicht möglich. Bei der Lungenuntersuchung zeigte die Patientin bibasilare Rasselgeräusche, bei der Herzuntersuchung eine regelmäßige Frequenz und einen regelmäßigen Rhythmus ohne Herzgeräusche oder Herztongallop sowie ein nicht druckschmerzhaftes Abdomen ohne Organvergrößerungen. An den Extremitäten wies sie beidseitig 2+ eindrückbare Ödeme ohne Trommelschlegelzehen auf. Ein großes Blutbild ergab eine Leukozytenzahl von 13.000/. $\mathrm{mm}^{3}$ und eine Thrombozytenzahl von 599.000/ $\mathrm{mm}^{3}$. Der Hämoglobinwert war unauffällig. Die Untersuchung relevanter grundlegender Stoffwechselparameter ergab folgende Ergebnisse: Creatinin 1,36 mg/dl (Ausgangswert 1,3) und Bicarbonat 35 mg/dl. Die
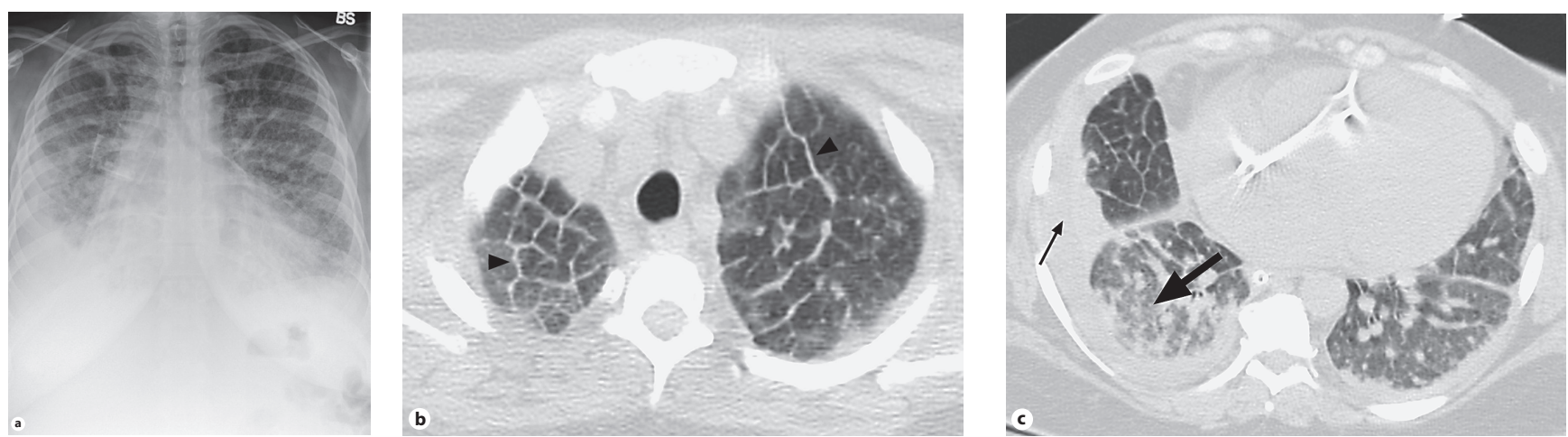

Abb. 1. (a) Der Röntgen-Thorax zeigt eine vergrößerte Herzsilhouette, einen mittelgroßen Pleuraerguss rechts, einen kleinen Pleuraerguss links sowie diffuse retikulärinterstitielle Veränderungen. (b, c) Das Thorax-CT zeigt eine diffuse, gleichmäßige Verdickung der interlobulären Septen (Pfeilspitzen), eine ungleichmäßige regionale Milchglastrübung (dicker Pfeil), einen kleinen Pleuraerguss rechts und eine Fissurverdickung (dünner Pfeil). 


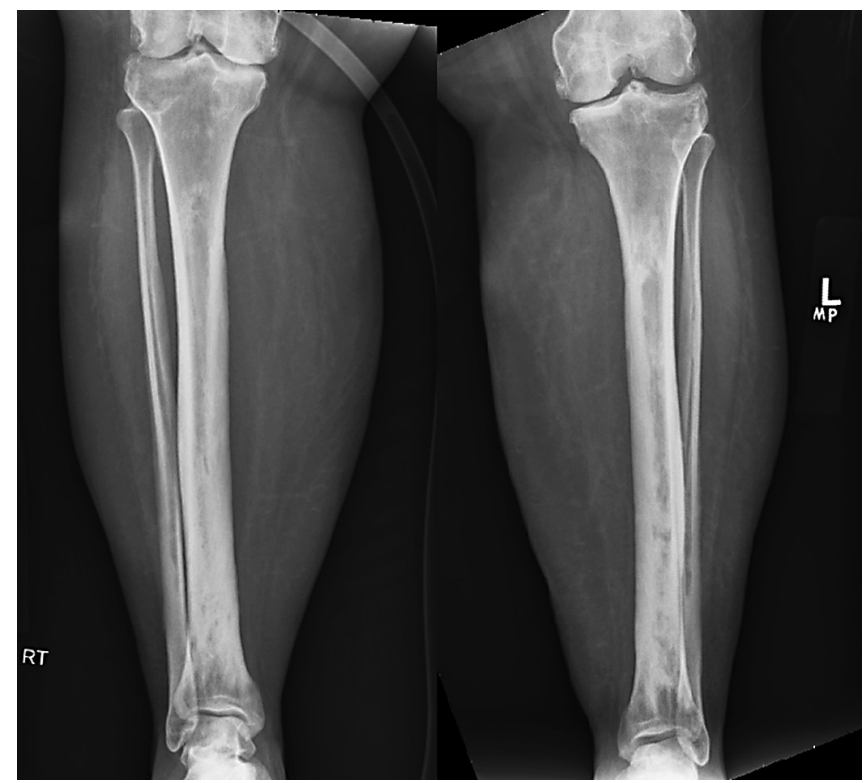

Abb. 2. Abb. 2. Die Röntgenaufnahme der unteren Extremitäten zeigt eine metadiaphysäre Osteosklerose mit Beteiligung beider Tibiae.

Urinanalyse war unauffällig. Die Analyse der arteriellen Blutgase (in der Notaufnahme mit der 50\% Venturi-Maske) ergab: $\mathrm{pH} 7,33$, pCO2 65, pO2 74 und Oxyhämoglobin 92\%.

Die bei der Vorstellung der Patientin durchgeführte Thorax-Röntgenaufnahme und Computertomographie (CT) sind in Abbildung
1 dargestellt. Ein 2D-Echokardiogramm ergab eine normale Größe des linken Ventrikels und systolische Funktion, eine leichte diastolische Dysfunktion und keine signifikanten Klappenanomalien. Anschließende Röntgenaufnahmen der unteren Extremitäten (Abb. 2) zeigten eine erhebliche Osteosklerose der Tibia und Fibula ohne Beteiligung der Epiphysen und eine weniger starke Femurbeteiligung.

Im Februar 2010 wurde die Patientin auf die Intensivstation aufgenommen, musste intubiert und künstlich beatmet werden. Trotz aggressiver Behandlung einer Volumenüberlastung vom 3.-16. Februar blieben die Anomalien in Bezug auf das Lungenparenchym weiter bestehen. Ein Pulmonalarterienkatheter ergab einen mittleren Lungendruck von 36 mmHg, einen Pulmonalarterien-Verschlussdruck von 15 mmHg und eine normale Herzleistung. Anschließend unterzog sich die Patientin einer chirurgischen Lungenbiopsie. Wie lautet Ihre Diagnose?

Übersetzung aus Respiration 2013;86:72-75, DOl: 10.1159/000348280

\section{Auflösung unter: \\ www.karger.com/blickdiagnose-kkp-4-2020}

Kontakt: Gustavo A. Heresi, The Cleveland Clinic Foundation, 9500 Euclid Avenue/A90, Cleveland, Ohio 44195, USA, heresig@ccf.org

\section{Aus der Praxis}

\section{Arztberuf im Wandel - welche Herausforderungen erwarten uns?}

Wie sieht die Zukunft im Arztberuf aus - vor allem im ambulanten Bereich? Darüber sprach Prof. Dr. Peter Rohmeiss beim Operation Karriere-Kongress in Heidelberg. Sein Rat: Bei aller Planung auch für Zufälle und spontane Entscheidungen offen zu bleiben.

«Uns steht ein gewaltiger Wandel bevor», erklärte Rohmeiss dem Publikum im Hörsaal der SRH Hochschule Heidelberg. Unter anderem seien die geplanten Krankenhausschließungen und der Hausarzt- und Fachärztemangel Themen, die den Arztberuf der Zukunft prägen werden. Doch ein Grund für Schwarzmalerei sei das nicht - Pessimismus habe es auch schon vor 20 Jahren gegeben. Man könne für die Zukunft daher einiges aus der Vergangenheit lernen. Rohmeiss begann mit einem Beispiel aus seinem Fachbereich, der Nephrologie: Während die Nierenheilkunde früher ein rein klinisches Fach gewesen sei, habe sie dann in Form von Dialysepraxen den Weg in den ambulanten Bereich gefunden. Nun kehren die niedergelassenen Fachärzte in die Kliniken zurück und übernehmen dort intensivmedizinische Leistungen, die sich die Kliniken nicht mehr leisten können. In anderen Bereichen, wie der Radiologie, gebe es eine ähnliche Entwicklung. In den Niederlanden sei es beispielsweise schon heute üblich, dass Zusammenschlüsse von niedergelassenen Fachärzten Kliniken ihre Leistungen zur Verfügung stellen.

\section{Klassische Niederlassung - ein Auslaufmodell?}

Rohmeiss wagte auch einen Blick in die Zukunft: Eine Konzentration im Bereich der Primärversorgung auf Krankenhäuser und Polikliniken werde es auch künftig nicht geben - es werde auch weiterhin dezentrale Strukturen und eine wohnortnahe Versorgung geben,

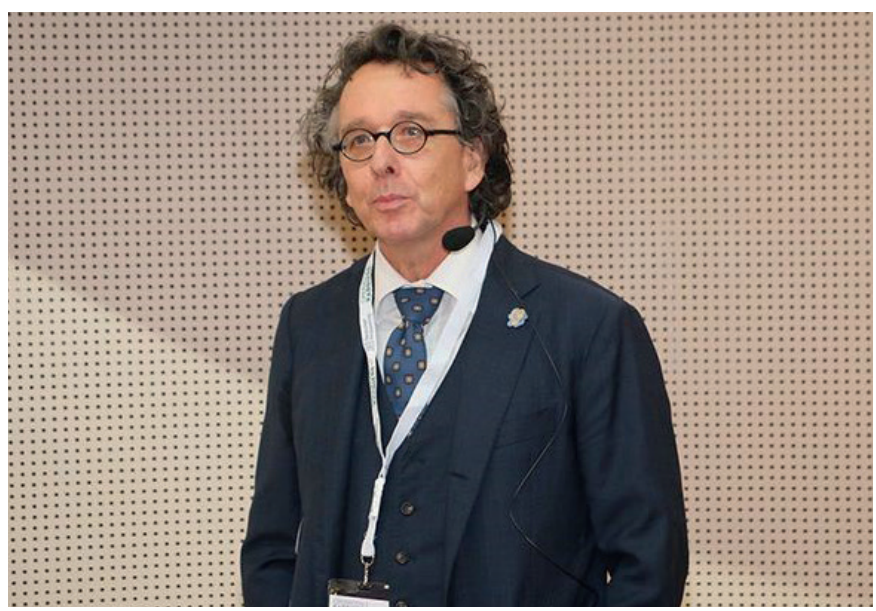

Wie sieht die Zukunft für Mediziner im ambulanten Bereich aus? Beim Operation Karriere-Kongress in Heidelberg wagte Prof. Dr. Peter Rohmeiss einen Blick in die Zukunft. @ Hanke 
prognostizierte er. Allerdings sei der klassische niedergelassene Arzt ein Auslaufmodell; künftig werde es keine Einzelkämpfer mehr geben. Schon jetzt gebe es viele verschiedene Kooperationsmöglichkeiten - von der Anstellung in einer Praxis bis zur Gemeinschaftspraxis.

Rohmeiss selbst ist Geschäftsführer der ze:roPRAXEN GbR - eines Praxenverbundes in der Rhein-Neckar-Region. Hier haben sich niedergelassene Ärzte verschiedener Fachgebiete zu einer größeren Struktur verbunden, die viele verschiedene Arbeitsmodelle ermögliche, erklärte er. Es gebe Teilzeitmodelle, Vollzeitmodelle, Praxisgründungen mit Eigenkapital oder als Juniorpartnerschaft oder auch Ärzte, die ein Leben lang angestellt arbeiten.

\section{Zwei neue Player auf dem medizinischen Spielfeld}

Die Zukunft werde vor allem von Teamwork und flacheren Hierarchien geprägt, prophezeite Rohmeiss. Im ambulanten Bereich gebe es das schon, aber auch in den Kliniken seien die aktuellen hierarchischen Strukturen nicht zukunftsfähig. Vor allem zwei neue Entwicklungen werden die Zusammenarbeit dabei drastisch verändern:

- Akademisierung der Pflegeberufe: Durch neue akademische Berufsbilder wie z.B. Physician Assistants (PAs), aber auch Bachelor, Master, Doktoren und sogar Professoren im Pflegebereich können ärztliche Leistungen künftig stärker delegiert werden.
- Künstliche Intelligenz als dritter Player: Außerdem werde es in wenigen Jahren immer mehr und immer bessere digitale Systeme geben, die Diagnosen und andere ärztliche Aufgaben übernehmen können. Schon jetzt gebe es gute Diagnoseprogramme: «ln drei Jahren wird es die ersten Google- und Amazon-Ärzte geben», prophezeite Rohmeiss.

«Patientendaten gehören nur in die Hände von Ärzten und Patienten und sollten nicht an Dritte weitergegeben werden - auch nicht über praktische Apps», forderte Rohmeiss in diesem Zusammenhang. Er beendete seinen Vortrag mit einem hoffnungsvollen Wunsch an die kommende Ärztegeneration: «Seien Sie offen - Ihnen steht eine faszinierende Zukunft bevor.»

Quelle: Operation Karriere-Kongress Heidelberg, 07.12.2019, Vortrag: «Der Arztberuf im Wandel - Perspektiven und Herausforderungen im ambulanten Bereich», Prof. Dr. med. Peter Rohmeiss, Geschäftsführer der ze:roPRAXEN, Schwetzingen.

OPERATION KARRIERE

\section{Fachtagung zur Medizin der Zukunft}

\section{Chronisch-entzündliche Erkrankungen maßgeschneidert behandeln}

\section{Individuelle Unterschiede erkennen und therapeutisch nutzen}

Chronische Entzündungserkrankungen wie Morbus Crohn, Schuppenflechte, Diabetes oder Rheuma unterscheiden sich nicht nur untereinander, sondern auch von Mensch zu Mensch. Bei diesen Unterschieden setzt die Präzisionsmedizin an: Sie will die individuellen Unterschiede zwischen einzelnen Betroffenen erkennen und diagnostisch und therapeutisch nutzbar machen. Ziel ist es u.a. messbare biologische Marker zu finden, die Voraussagen erlauben,

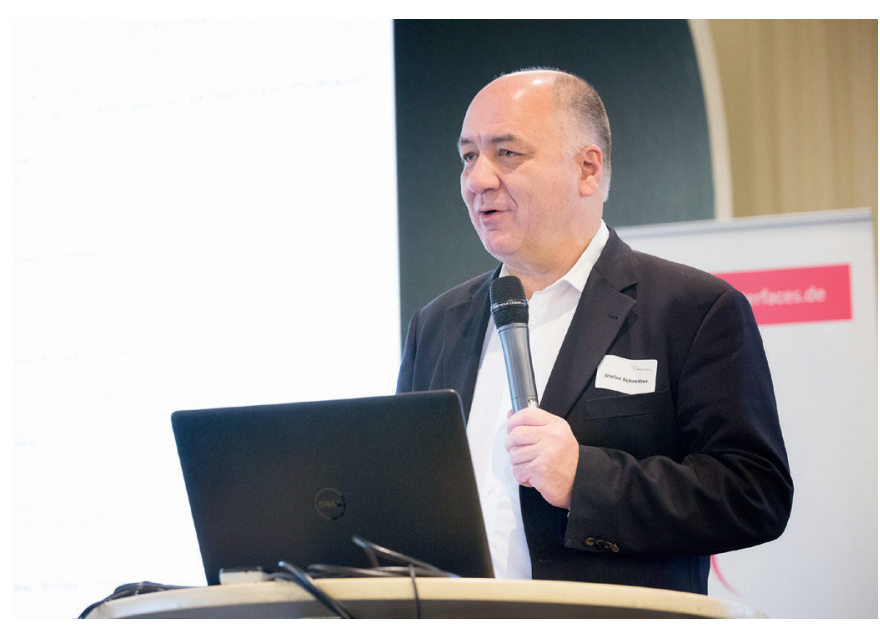

Prof. Stefan Schreiber. ๑ C. Kloodt, PMI welche maßgeschneiderten Therapien für jeden individuellen Fall sinnvoll eingesetzt werden können.

Die Präzisionsmedizin war das zentrale Thema des 7. Internationalen Symposiums «Inflammation Medicine - From Bench to Bedside», zu dem das Exzellenzcluster «Precision Medicine in Chronic Inflammation» (PMI) Mitte Februar nach Hamburg einlud. Auf der Fachtagung diskutierten rund 250 internationale Wissenschaftlerinnen und Wissenschaftler aus Klinik und Grundlagenforschung die neuesten Fortschritte in der Entzündungsmedizin. Dabei

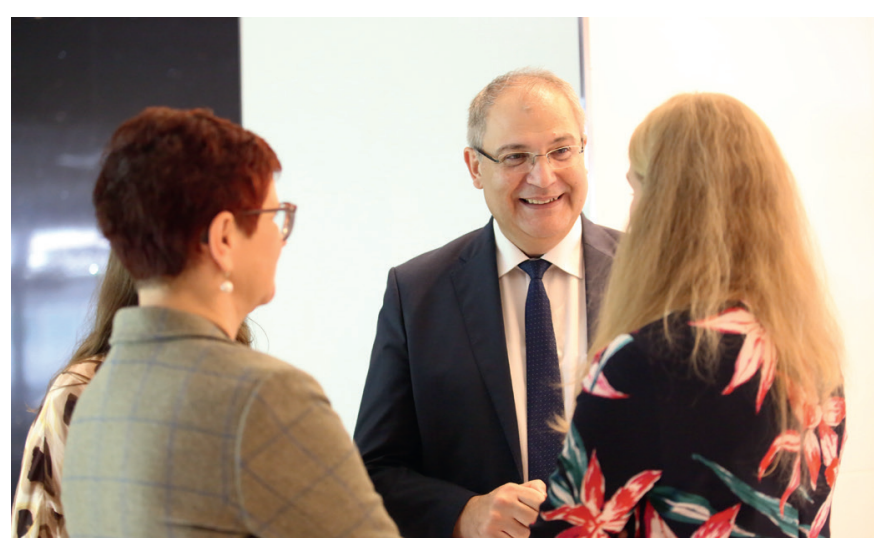

Prof. Diamant Thaçi im Gespräch. (C) C. Kloodt, PMI

Tagungsbericht zum 7. Internationalen Symposium «Inflammation Medicine From Bench to Bedside» vom 17.-18. Februar 2020 in Hamburg. 
tauschten sich die Experten über den Stand der Forschung zur Genetik von autoinflammatorischen Erkrankungen, zum Mikrobiom und seinem Stellenwert in der Entstehung von chronischen Entzündungsprozessen sowie über den aktuellen Forschungsstand zu häufigen chronisch-entzündlichen Erkrankungen aus. Ein Angebot speziell für junge Forschende waren die Posterpräsentationen sowie ein Workshop zum wissenschaftlichen Publizieren. «Die Nachwuchsförderung ist eine wichtige Mission für uns», betont Mitorganisator Professor Diamant Thaçi, Dermatologe und Leiter des Exzellenzzentrums Entzündungsmedizin am UKSH, Campus Lübeck.

\section{Zielvorgabe ist die Erforschung der Präzisionsmedizin}

«Bei chronischen Entzündungserkrankungen haben wir das Problem, dass ein Medikament bei einigen Erkrankten gut wirkt und bei anderen nicht», erklärt Cluster-Sprecher Professor Stefan Schreiber, Direktor des Instituts für klinische Molekularbiologie (IKMB) der Christian-Albrechts-Universität zu Kiel (CAU) und Direktor der Klinik für Innere Medizin I am UKSH, Campus Kiel. «Aktuell können wir das überwiegend nur durch Ausprobieren herausfinden». Bereits vor Therapiebeginn vorherzusagen, welche Behandlung bei der individuellen Patientin oder dem individuellen Patienten am erfolgreichsten sein wird, sei daher ein wichtiges Ziel der Forschung, so schreiber weiter.
Gezielt an praktische Ärzte richtete sich das klinische Symposium «Individualisierte Medizin für chronisch entzündliche Erkrankungen in der Pulmologie, Rheumatologie, Dermatologie und Gastroenterologie». Hier gaben internationale und deutsche Expertinnen und Experten Einblicke in die neuesten Therapiemöglichkeiten entzündlicher Erkrankungen und deren Wirkmechanismen. «Es ist selten so, dass eine Entzündung in einem Organ stecken bleibt», begründet Thaçi den interdisziplinären Ansatz. Es gelte in verschiedenen Organen die Triggerfaktoren und Prädiktoren für Erkrankungen früher zu erkennen und auf dieser Basis Therapien zu entwickeln, die dem Patienten mehr Nutzen und weniger Risiken bei der Behandlung bringen.

Noch gehört die Präzisionsmedizin zwar nicht zur Standardbehandlung, doch die Forschung nimmt hier Fahrt auf. Thaçi sieht in ihr viel Potenzial: «Für die Behandlung von chronischen Entzündungen sind bereits einige neuartige Medikamente auf den Markt gekommen, mit denen wir die Behandlung noch präziser als bisher an den zugrundeliegenden molekularen Mechanismen der individuellen Betroffenen ausrichten können.» 